\title{
Innenstadt und stationärer Einzelhandel - ein unzertrennliches Paar? Was ändert sich durch den Online-Handel?
}

\author{
Martina Stepper ${ }^{1}$
}

Eingegangen: 30. März 2015 / Angenommen: 7. März 2016 / Online publiziert: 22. März 2016

(C) Springer-Verlag Berlin Heidelberg 2016

\begin{abstract}
Zusammenfassung Stationärer Einzelhandel und (Innen-) Stadt sind räumlich betrachtet eng miteinander verflochten. Durch die zunehmende Relevanz des Internets und insbesondere des damit verbundenen Online-Handels im Alltag der Menschen verliert der physische Raum als konstantes Bindeglied aktuell jedoch immer mehr an Bedeutung. Vor dem Hintergrund der allgemeinen Zeitknappheit und den Vorzügen, welche der Online-Handel bietet, werden immer mehr Produkte online und nicht im stationären Einzelhandel erworben. Dies schlägt sich auch in der Umsatzentwicklung nieder. Während der stationäre Einzelhandel in den letzten Jahren nahezu stagnierende Umsätze zu verzeichnen hat, sind die Wachstumsraten im Online-Handel immens. Bis 2025 soll der Online-Anteil am gesamten Einzelhandelsumsatz auf rund $15 \%$ anwachsen. Dies bleibt nicht ohne Folgen für innerstädtische Einzelhandelslagen. Es wird - abhängig von ihrer Lokalisierung in räumlich-strukturell begünstigten oder benachteiligten Städten - sowohl Gewinner als auch Verlierer geben. Um die Folgen für die davon betroffenen Räume möglichst gering zu halten, sollten die Möglichkeiten, welche die zunehmende Digitalisierung bietet, als Chance für die Attraktivitätssteigerung der innerstädtischen Einzelhandelslagen genutzt werden.
\end{abstract}

Schlüsselwörter Innenstadt · Stationärer Einzelhandel · Online-Handel · Innerstädtische Einzelhandelslagen

Dr.-Ing. Martina Stepper

Martina.Stepper@ru.uni-kl.de

1 Lehrstuhl Stadtplanung, TU Kaiserslautern, Pfaffenbergstr. 95, 67663 Kaiserslautern, Deutschland

\section{Inner city and Stationary Retail-an Inseparable Couple? What are the Implications of Online Trading?}

Abstract Stationary retail and inner cities have a tight interconnection on the spatial level. Due to the increasing importance of Internet and online trading in everyday life, however, the physical space as a constant link currently becomes less important. Against the background of general lack of time and the benefits, which online trading provides, more and more products are purchased online. This is also reflected in the trading volume. While counter retail has made almost stagnant sales in recent years, the growth rates in online commerce are immense. The online share of total retail sales is expected to grow to around $15 \%$ by 2025 . This is not without consequences for urban retail locations. There will be - depending on their localization in cities that are favored or disadvantaged in spatial-structural termsboth winners and losers. To keep the impact on affected areas as low as possible, the opportunities offered by the increasing digitization should be used as an opportunity for increasing the attractiveness of inner-city retail locations.

Keywords Inner city - Stationary retail - Online trading · Inner-city retail locations

\section{Die Beziehung von stationärem Einzelhandel und (Innen-) Stadt - eine Einführung}

Stationärer Einzelhandel und Innenstadt stehen in einem besonderen Abhängigkeitsverhältnis. Ihr Erfolg - gemessen an Prosperität und Attraktivität - hängt wesentlich von dem jeweils anderen Faktor ab, da sie zusammen die lokalen Rahmenbedingungen prägen. 
Die gegenseitige Bedeutung von stationärem Einzelhandel und Innenstadt füreinander zeigt sich in dreierlei Hinsicht:

1. Strukturelle Bedeutung: Zum einen haben die örtlichen Einkaufsmöglichkeiten, d. h. ihre Qualität und Quantität, großen Einfluss auf das Image und die damit verbundene Anziehungskraft einer Innenstadt. So sind nach einer repräsentativen Befragung der CIMA für $66 \%$ der Befragten die Einkaufsmöglichkeiten ausschlaggebend für die Attraktivität einer Innenstadt (Mensing 2010: 30). Zum anderen zählen der Bedeutungsüberschuss der Innenstadt gegenüber anderen Räumen sowie ihre Funktions- und Nutzungsmischung zu den wesentlichen Standortfaktoren des stationären Einzelhandels. Sie garantieren ihm die benötigte hohe Frequenz an Besuchern, welche für die Einzelhändler potenzielle Kunden darstellen. Dies spiegelt sich auch in den Expansionszahlen der Händler wider. $70 \%$ der Händler wollen in die A- und/oder B-Lagen der Innenstädte expandieren. Insbesondere Händler mit Verkaufsflächen unter 100 qm fragen die A-Lagen aufgrund der kleinteiligen Baustruktur nach. 92\% der Händler geben diese Lagen als Favoriten an (EHI Retail Institute GmbH 2015: 8).

2. Räumlich-gestalterische Bedeutung: Einerseits werden innerstädtische Baustrukturen und somit das Erscheinungsbild der Innenstädte wesentlich durch den stationären Einzelhandel mit seinen dominanten Handelsbauten - wie Warenhäusern und Shopping-Centern-sowie den in den Erdgeschoss-Zonen vorherrschenden Ladenlokalen bestimmt. Ca. $63 \%$ der Gesamteinzelhandelsflächen in Deutschland befinden sich im innerstädtischen Bereich und an integrierten Standorten (Stand 2012) (Nitt-Drießelmann 2013: 21). Bis zu 2 Mrd. $€$ investieren Händler gemäß dem Handelsverband Deutschland (HDE) jedes Jahr in Neu- und Umbaumaßnahmen sowie den Denkmalschutz (HDE 2014a: 5). Andererseits bietet die Innenstadt mit ihrer "schönen urbanen Verpackung“1 (Kunzmann 2012: 159) das notwendige individuelle und identifikationsstiftende Profil für den stationären Einzelhandel, welches von den heutigen Konsumenten nachgefragt wird (Laux 2012: 41).

3. Bedeutung in ökonomischer Hinsicht: Einerseits ist der stationäre Einzelhandel mit knapp 3 Mio. Beschäftigten im Einzelhandel insgesamt - Tendenz steigend - (HDE 2015a: 7) ein wichtiger Arbeitgeber für die (Innen-) Stadt sowie ein Ort der Kommunikation und des Austauschs. Andererseits profitieren die Städte von den Gewerbesteuereinnahmen eines florierenden innerstädtischen Einzelhandels.

\footnotetext{
${ }^{1}$ Kunzmann beschreibt hiermit die wachsende Bedeutung von „Design“ im Sinne von Architektur, Werbung und Straßenmöblierung im öffentlichen Raum.
}

Diese wechselseitige Bedeutung von stationärem Einzelhandel und Innenstadt füreinander bringt Potenziale, aber auch Risiken mit sich. Solange sich beide Seiten positiv entwickeln, können sie voneinander profitieren. Was aber passiert, wenn sich eine Seite zum Nachteil der anderen entwickelt? Vom Handel geht in dieser Hinsicht eine wesentlich größere Gefahr aus als von der Innenstadt, da er deutlich kürzere Veränderungszyklen ${ }^{2}$ aufweist. Diese liegen bei ca. 7 bis 10 Jahren, während die städtischen Strukturen mit Veränderungszyklen von 25 Jahren und mehr deutlich beständiger sind (Reink 2014: 20). Der stationäre Einzelhandel befindet sich folglich - bedingt durch die sich wandelnden gesellschaftlichen, politischen und technischen Rahmenbedingungen - in einem beständigen strukturellen Wandlungsprozess hinsichtlich seiner Lokalisierung, Betriebsformen, Sortimentsbreite und -tiefe, Marketingkonzepten etc. Neben diesen zum Teil tief greifenden Veränderungen bzw. Neuerungen schien einzig die Abhängigkeit von einem möglichst attraktiven physischen Standort, zu dem die Innenstadt bis heute zählt, ${ }^{3}$ eine unabänderliche Konstante zu sein. Diese „topologische Konstante“, wie Christ (2014: 67) sie bezeichnet, wird durch den rasant wachsenden Online-Handel seit einigen Jahren hart auf die Probe, wenn nicht sogar grundsätzlich in Frage gestellt. So bleibt die Erweiterung des Absatzmarktes in den virtuellen Raum hinein nicht ohne Folgen für den lokalen Einzelhandel und einen seiner wichtigsten Standorte, die Innenstadt. Insbesondere der innerstädtische Einzelhandel wird aufgrund seines internetaffinen Sortiments zunehmend vom Online-Handel betroffen sein (s. Kapitel 3.2).

Ziel des Beitrages ist es, die möglichen Auswirkungen des wachsenden Online-Handels auf den stationären Einzelhandel in zwei später definierten Innenstadttypen - „den Gewinnern“ und „den Verlierern“ der dargestellten Entwicklung - aufzuzeigen.

Zur begrifflichen Klärung werden zunächst (Kapitel 2) Innenstadt und stationärer Einzelhandel definiert. Das nachfolgende Kapitel 3 gibt einen Überblick über die wachsende Bedeutung des Online-Handels. Dessen Bedeutungszuwachs wird am Nutzungsinteresse der Bevölkerung sowie der Umsatzentwicklung festgemacht. Die Betroffenheit des innerstädtischen Einzelhandels von den Umsatz-

\footnotetext{
${ }^{2}$ Veränderungszyklen sind strukturelle Wandlungsprozesse, die periodisch auftreten. Im Einzelhandel haben sich beispielsweise die Renovierungszyklen der Ladenlokale in den letzten Jahren aufgrund des hohen Wettbewerbsdrucks auf durchschnittlich sieben Jahre verkürzt (Stand 2010) (KPMG AG Wirtschaftsprüfungsgesellschaft/EHI Retail Institute 2012: 25). Städtebauliche Strukturen wie die Innenstadt sind vergleichsweise träge und verändern sich deutlich langsamer (Schubert 2008: 33)

${ }^{3}$ Gemäß einer aktuellen Untersuchung in 62 Städten unterschiedlicher Größenklassen geben die befragten 33.000 Innenstadtbesucher den Innenstädten im Durchschnitt die Schulnote drei plus (IFH Köln 2015b: 1).
} 
verschiebungen hin zum Online-Handel wird anhand verschiedener Studienergebnisse belegt. Als Ergebnis (Kapitel 4) werden mögliche Konsequenzen für die innerstädtischen Einzelhandelslagen prognostiziert und darauf aufbauend in Kapitel 5 ein Ausblick gegeben.

Methodische und inhaltliche Basis des vorliegenden Artikels ist eine umfangreiche Sekundäranalyse bestehender Studien, welche im Rahmen der Dissertation der Autorin (Stepper 2015) durchgeführt worden ist.

\section{Innenstadt und stationärer Einzelhandel - Begriffsbestimmungen}

Die Innenstadt wird häufig mit Begriffen wie Altstadt, City, (städtisches) Kerngebiet, Fußgängerzone, Kernstadt, Stadtmitte oder auch Stadtkern gleichgesetzt, welche von ihrer Bedeutung her jedoch nur bedingt synonym verwendet werden können und sich oftmals räumlich überlagern (Wietzel 2007: 13 ff.). Die räumliche Abgrenzung für die Entwicklung des Innenstadtmodells erfolgt daher analog dem Vorgehen von Wietzel auf der Basis des Lagetypenmodells des BBR (2010) sowie nach den Ausführungen von Ebert/Zlonicky (1990: 91) (s. Abbildung 1).

Demnach umfasst die Innenstadt idealtypischerweise

- das Gebiet der Altstadt als dem ältesten Teil bzw. der historischen Keimzelle der Stadt,

- die Fußgängerzone, welche die bekannteste Ausprägung der innerstädtischen Einzelhandelslagen ${ }^{4}$ darstellt,

- den Bahnhof als zentralen Verkehrsknotenpunkt und seine nähere Umgebung,

- die City, welche den ,zentrale[n] Standort mit der höchsten, auf tertiärer Nutzung basierenden Zentralität““ (Hotzan 2004: 173) bezeichnet sowie

- die angrenzenden Ergänzungsgebiete, die auch Cityrand oder Citymantel genannt werden und zugunsten von Wohnnutzungen eine geringere Intensität und Dichte der innerstädtischen Funktionen aufweisen (Wietzel 2007: 14 ff.).

Der Einzelhandel ist eine der wichtigsten Leitfunktionen ${ }^{5}$ der Innenstadt. Bestimmendes Merkmal des Einzelhandels ist, dass der Verkauf von Waren und Dienstleistungen ohne Zwischenhändler direkt vom Einzelhändler an den Konsumenten als Letzt- bzw. Endverbraucher erfolgt (Weers-Hermanns 2007: 43). Der stationäre Einzelhandel als Teilgebiet des Einzelhandels findet im Gegensatz zum

\footnotetext{
${ }^{4}$ Siehe dazu die nachfolgende Definition.

${ }^{5}$ Die Innenstadt ist neben dem Einzelhandel im Wesentlichen durch vier weitere charakteristische Funktionen geprägt: „Wohnen“, „Dienstleistung und Verwaltung“, „Arbeitsplätze“ sowie „Kultur, Freizeit und Gastronomie“ (Wüstenrot Stiftung 2008).
}

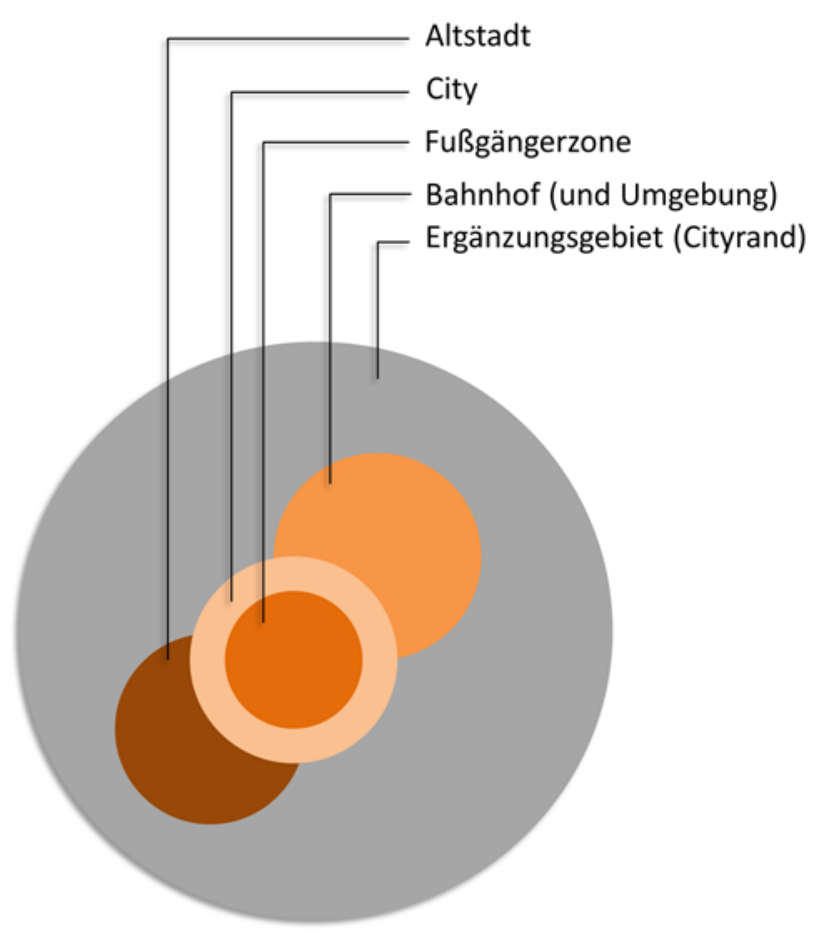

Abb. 1 Innenstadtmodell. (Quelle: Eigene Darstellung auf der Basis von Wietzel 2007: 17)

ambulanten Handel (welcher nach dem Bringe- oder Treffprinzip erfolgt) örtlich gebunden an einem festen, räumlich unflexiblen Standort statt (Müller-Hagedorn 1998: 45). Klassischer Verkaufsraum ist ein physisches Ladenlokal.

Besonders betrachtet wird hier der stationäre Einzelhandel, welcher in der Innenstadt lokalisiert ist, genauer die innerstädtischen Einzelhandelslagen. $\mathrm{Zu}$ diesen zählen die Bereiche der Innenstadt, die funktional vom stationären Einzelhandel geprägt sind und somit zentrale Versorgungsbereiche der Stadt darstellen. Räumlich umfasst der Begriff die privaten Geschäftsräume der Einzelhändler sowie die angrenzenden öffentlichen Zuwege, die Fußgängerzonen, Straßen und (Markt-) Plätze in der Innenstadt (Stepper 2015: 9).

\section{Der virtuelle Raum als erweiterter Handelsraum - Die Bedeutung des Online- Handels}

Seit dem Beginn des klassischen (Katalog-) Versandhandels Ende des 19. Jahrhunderts (Eli/Laumer 1969: 16), als dessen Fortsetzung mit anderen technischen Mitteln der OnlineHandel verstanden werden kann, ist der Handel nicht mehr auf einen festen lokalen Standort beschränkt. Der physische Raum wird um den virtuellen Raum erweitert. Dies erhöht den Wettbewerbsdruck für den stationären Einzelhandel, birgt aber auch Potenziale (s. Kapitel 5). 


\subsection{Die Einstellung der Bevölkerung zum Online- Handel}

Eine knappe Mehrheit der deutschen Bevölkerung (45 Mio. Menschen) erwirbt Waren oder Dienstleistungen für private Zwecke (auch) über das Internet (Stand: 2013). Damit kaufen $76 \%$ der Internetnutzer ab 10 Jahren über das Internet ein. Dies ist ein Anstieg um rund 30\% im Vergleich zum Jahr 2008. Seinerzeit gab es rund 35 Mio. private OnlineKäufer (Statistisches Bundesamt 2014: 1).

$\mathrm{Zu}$ den treibenden Faktoren der Entwicklung zählt weniger die wachsende Zahl der Online-Käufer an der Gesamtbevölkerung als vielmehr der wachsende Anteil der intensiven Online-Käufer, d. h. derjenigen Online-Käufer, welche mindestens zehn Transaktionen innerhalb der letzten 12 Monate online getätigt haben. Während im Jahr 2004 lediglich $6 \%$ der Gesamtbevölkerung zu den intensiven Online-Käufern zählten, waren es 2012 bereits mehr als ein Fünftel (21\%) (Köcher 2012: 6).

Die hohe Akzeptanz des Online-Handels, welche sich in stetig steigenden Nutzungsraten und Umsatzsteigerungen (s. Kapitel 3.2) widerspiegelt, geht vor allem auf die verbesserten technischen Rahmenbedingungen zurück, welche den Einkauf über das Internet in der heutigen Form erst möglich gemacht haben. Dazu zählen:

- der voranschreitende Netzausbau (räumliche Erschließung, steigende Bandbreiten),

- sinkende Hardware-, Zugangs- und Nutzungskosten sowie

- insbesondere die verbesserte Nutzbarkeit (usability) digitaler Medien. Sie sind effektiver, effizienter, sicherer und funktionaler sowie in ihren Anwendungsmöglichkeiten begreifbarer geworden, da sich Prinzipien beispielsweise für die einheitliche Benutzbarkeit von Internetseiten durchgesetzt haben (Alby 2008: 10) . Auch der Online-Handel ist professioneller geworden. Produkte werden immer aufwendiger präsentiert, neue Bezahlsysteme wie PayPal verbessern die Sicherheit, und die Ausweitung neuer, auf den Konsumenten abgestimmte Lieferkonzepte erhöhen den „ConvenienceFaktor“ (Doplbauer 2015: 11). Des Weiteren hat die Einführung attraktiverer und einfach zu bedienender Endgeräte, wie Tablet-PCs und Smartphones, die Nutzbarkeit erhöht (Malaka/Butz/Hussmann 2009: 44).

Infolge dieser verbesserten Rahmenbedingungen sind immer mehr Haushalte mit Informations- und Kommunikationstechnologien (IuK-Technologien) ausgestattet. Gemäß der ARD/ZDF-Online-Studie nutzten 2014 95\% einen Inter- netzugang über einen Computer oder einen Laptop sowie $57 \%$ über ein Smartphone (ARD/ZDF-Medienkommission 2015). Ferner verbessert sich das digitale Potenzial der Nutzer. Dieses bezeichnet die Fähigkeit, digitale Medien entsprechend den eigenen Bedürfnissen selbstständig zu nutzen und mit ihnen umgehen zu können.

Zum anderen hat der Online-Handel seinen ganz eigenen Reiz entwickelt, da er - wie Abbildung 2 zeigt - viele Vorzüge gegenüber dem stationären Einzelhandel aufweist.

Die Vorzüge von Online-Shopping bestehen zusammengefasst in einer hohen Convenience-Orientierung (Unterstützung individueller Bequemlichkeit) und einer großen Individualität. Beide Merkmale zählen zu den zentralen Bedürfnissen bzw. den wichtigsten Anforderungen heutiger Konsumenten. Laut der InterOne $\mathrm{GmbH}$ (2011: 7) ist die Convenience-Orientierung sogar wichtiger als der Preis; nur für 22 bis $36 \%$ der befragten Konsumenten ist der Preis das Hauptmotiv für den Kauf im Internet.

Einkaufen über das Internet bietet infolge des geringen Zeitaufwands, der niedrigen Informationskosten sowie des hohen Grads an Angebots- und Preistransparenz einen hohen Komfort. Durch die Zeit- und Ortsungebundenheit, die hohe Aktualität des Sortiments sowie die große Sortimentsauswahl ist der Online-Einkauf bequem und lässt dem Einzelnen viele Freiräume. Durch einen ausgeprägten persönlichen Zuschnitt der Produkte kommt er zudem dem Wunsch nach Individualität nach.

Insgesamt tragen der hohe Bequemlichkeitsgrad sowie die Individualität dazu bei, dass Online-Shopping nicht nur für „Konsum-Interessierte“, sondern auch für „ShoppingMuffel" attraktiv ist; vorausgesetzt sie bringen das digitale Potenzial mit. So gaben im Rahmen einer Befragung der Deutschen Post DHL im Jahr 2011 44\% der Befragten an, dass sich ihre persönliche Lebensqualität durch die Möglichkeit des Online-Einkaufs verbessert hat, weil der Spaß beim Einkaufen steigt, der Online-Einkauf Stress reduziert und das Zeitmanagement verbessert (Deutsche Post DHL 2012: 27, 29).

\subsection{Umsatzentwicklung des Online-Handels}

Ein wichtiger Indikator für das wachsende Nutzungsinteresse der Konsumenten am Online-Handel sowie für seine Bedeutung gegenüber dem stationären Einzelhandel ist seine Umsatzentwicklung. Eindeutige Aussagen zur Umsatzentwicklung sind jedoch schwierig, da die Angaben zu den absoluten Umsätzen und den Steigerungsraten infolge der unterschiedlichen Erhebungsgrundlagen

\footnotetext{
${ }^{6}$ Mit der verbesserten Nutzbarkeit digitaler Medien geht eine höhere Zufriedenheit bei der Nutzung einher. Ihre Attraktivität für die Nutzer steigt (Stepper 2015: 141).
} 
Abb. 2 Die zentralen Stärken des Online-Shopping aus Konsumentensicht. (Quelle: Eigene Darstellung)
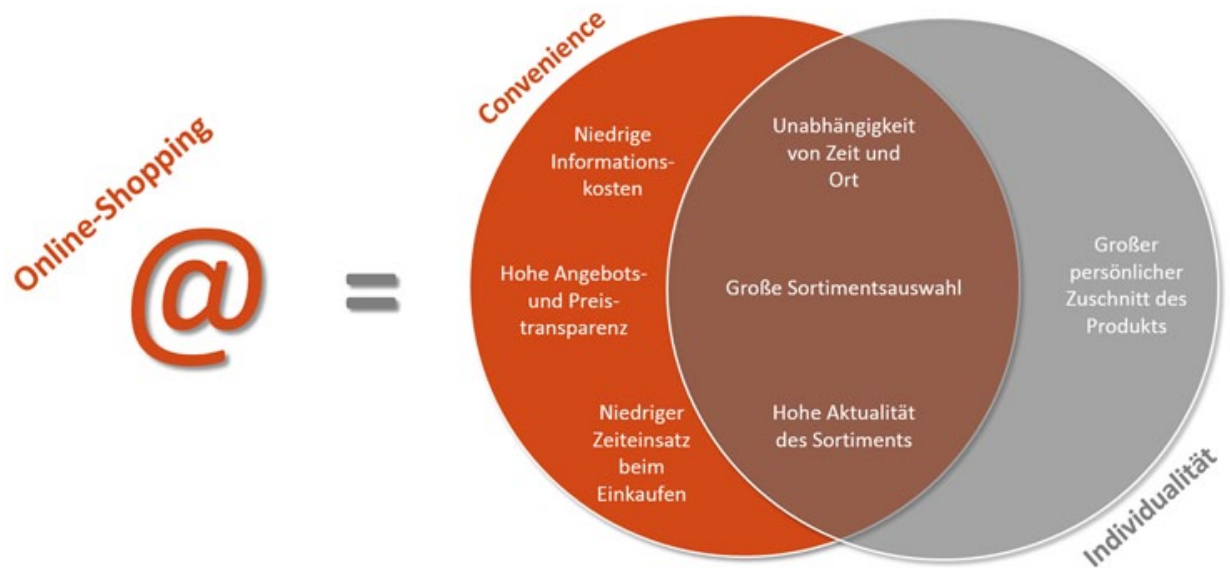

der Institute variieren. ${ }^{7} 2014$ wurden - je nach Quelle $^{8}$ - zwischen 37,1 und 42 Mrd. $€$ im Online-Handel erwirtschaftet. Die Angaben zu den Wachstumsraten von 2013 auf 2014 schwanken zwischen 6,5 und 11,3\% (HDE 2015b; bevh 2015; IFH Köln 2015a). ${ }^{9}$

Deutlich wird dennoch, dass sich die Umsätze im OnlineHandel eindeutig positiv entwickeln; seine Bedeutung wächst. Demgegenüber stehen nahezu stagnierende Umsätze im stationären Einzelhandel (Dorn 2015: 35), die seit dem Jahr 2012 eindeutig auf die wachsende Konkurrenz durch den Online-Handel zurückzuführen sind (Bender 2013). So ist der stationäre Einzelhandel von 2013 auf 2014 lediglich um 1,7\% gewachsen; seit 2009 lagen die Zuwachsraten konstant unter 2,5\% und waren in einigen Jahren sogar negativ (HDE 2015c: 7). Allerdings beträgt das umgesetzte Volumen immer noch ein Vielfaches des

\footnotetext{
${ }^{7}$ Der HDE (Handelsverband Deutschland e. V.) und der bevh (Bundesverband E-Commerce und Versandhandel Deutschland e. V.) sind die beiden größten deutschen Einzelhandelsverbände. Während der HDE vom stationären Einzelhandel geprägt ist, vertritt der bevh den Online- und Versandhandel. Diese unterschiedliche Ausrichtung spiegelt sich auch in den unterschiedlichen Erhebungsgrundlagen wider. Dies wird anschaulich in einem Artikel der Neuen Mediengesellschaft Ulm mbH dargestellt (2014). Die Datenerhebung des IFH Köln basiert auf Angaben der Anbieter bzw. Umsatzangaben zu den einzelnen Unternehmen sowie statistischen Daten zu Handelsunternehmen, also ebenfalls anbieterseitigen Informationen. Die Ergebnisse werden anhand vielfältiger eigener Befragungen und mithilfe des IFH Brancheninformationssystems BIS, welches kontinuierliche Marktdaten aus 60 Konsumgütermärkten und 500 Warengruppen umfasst und damit $90 \%$ des gesamten Handelsvolumens abdeckt, sowie aller verfügbaren Quellen überprüft und plausibilisiert. Alle Marktvolumenangaben sind - wie beim bevh - Werte zu Endverbraucherpreisen, d. h. inklusive Mehrwertsteuer. Dementsprechend ähnlich sind die Zahlen vom IFH Köln sowie vom bevh.

${ }^{8}$ Für 2014 wurden folgende Umsätze im Online-Handel ermittelt: HDE: 37,1 Mrd. €, bevh: 41,9 Mrd. € und IFH Köln: 42 Mrd. $€$.

${ }^{9}$ Angaben zur Wachstumsrate von 2013 auf 2014: HDE: 6,5\%, bevh: 7\%, IFH Köln: 11,3\%.
}

Online-Handels. Im Jahr 2014 wurden im gesamten Einzelhandel 457 Mrd. $€^{10}$ umgesetzt (IFH Köln 2014a: 5).

Werden die Zahlen der Gesellschaft für Konsumforschung (GfK) zugrunde gelegt, lag der Anteil des Online-Handels am gesamten Einzelhandelsumsatz 2014 trotz seiner deutlichen Zuwächse bei lediglich $8,5 \%$. Bis 2025 prognostiziert das Institut einen weiteren deutlichen Anstieg. Der Online-Anteil am gesamten Einzelhandelsumsatz soll bis dahin auf rund $15 \%$ anwachsen (Doplbauer 2015: 13). Damit würden die Umsatzverschiebungen vom stationären Einzelhandel zum Online-Handel standortrelevant. So gilt ein Handelskanal bereits ab einer Umsatzverschiebung von $10 \%$ landesplanerisch und städtebaulich als nicht mehr verträglich für die anderen Kanäle, auch wenn diese sogenannte „10-Prozent-Schwelle“ zur rechtlichen Bewertung der Umsatzumlenkungen bisher nicht gesetzlich vorgegeben ist (Wolf 2012: 124).

Auch das Institut für Handelsforschung (IFH Köln) ist von den schädlichen Auswirkungen des prognostizierten höheren Anteils des Online-Handels am Gesamteinzelhandelsumsatz auf den innerstädtischen Einzelhandel überzeugt. Es prognostiziert den stationären Einzelhändlern langfristig einen Umsatzrückgang um rund $10 \%$ bis zum Jahr 2020 (AFP 2014).

Insbesondere die Innenstädte werden von den Umsatzverschiebungen betroffen sein. So gilt es zu bedenken, dass es sich bei den genannten Umsatzanteilen um Durchschnittswerte aller Sortimentsbereiche handelt. Vor allem die innenstadtrelevanten Sortimente, welche sich durch eine hohe Anziehungskraft für die Innenstadtbesucher und infolgedessen durch eine zentrumsstärkende und -bewahrende Wirkung auszeichnen (Heitfeld-Hagelgans 2012: 24), sind - wie Abbildung 3 zeigt - insbesondere auch für den Verkauf über das Internet geeignet.

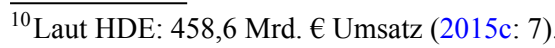


Abb. 3 Internetaffinität der in der Innenstadt vertriebenen Produkte. (Quelle: Eigene Darstellung)

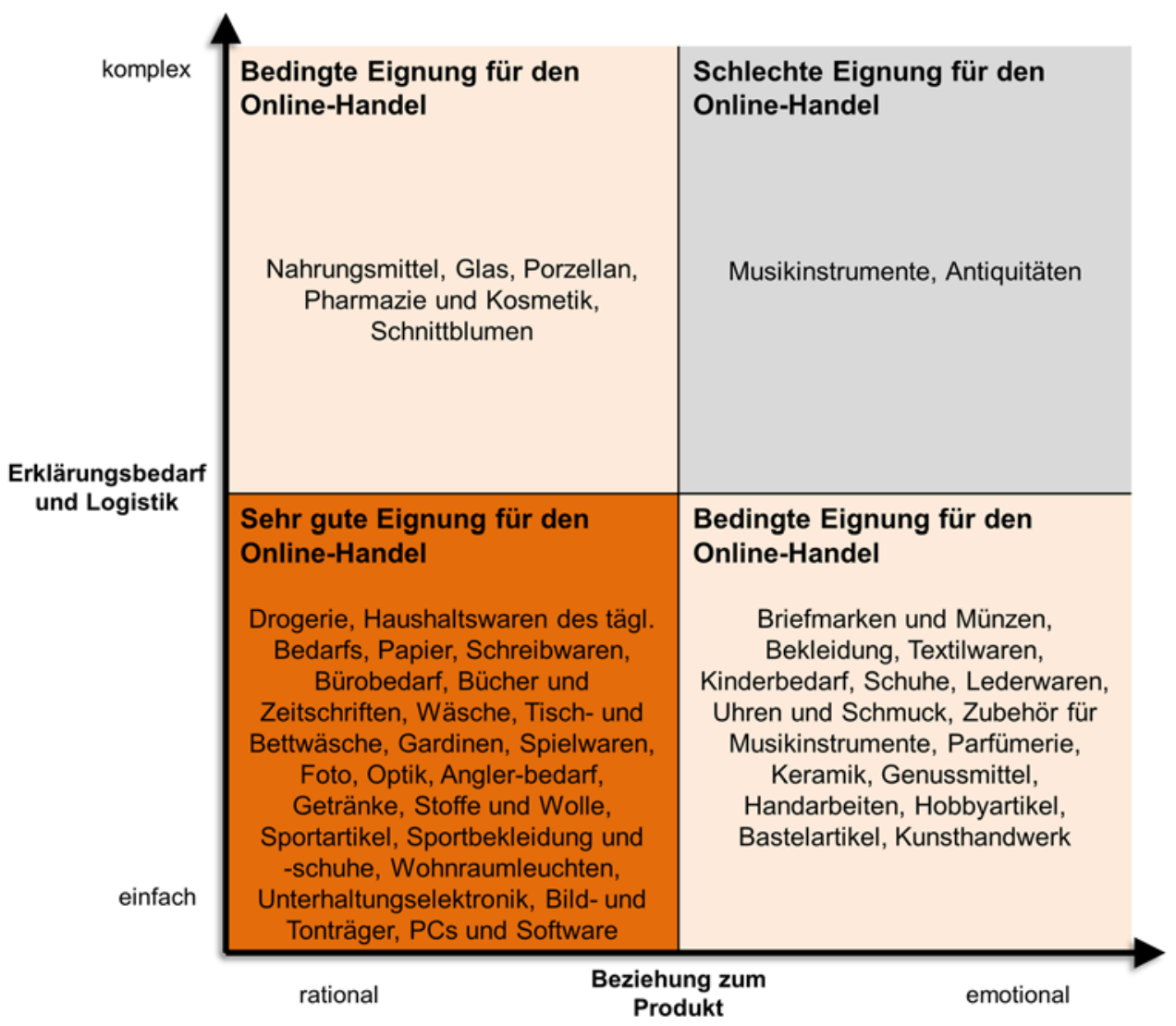

Bestimmende Faktoren für die Eignung eines Artikels für den Online-Handel - d. h. Kriterien, nach denen der Kunde den Nutzen bemisst, einen Kauf über das Internet abzuschließen ${ }^{11}$ bzw. nach denen der Händler einen Mehrwert im Online-Angebot der Ware oder Dienstleistung sieht - sind im Wesentlichen (IFH Retail Consultants GmbH 2012):

- die Komplexität des logistischen Vorgangs, welche nicht nur durch die Transportentfernung bestimmt wird, sondern daneben auch von den Eigenschaften des bestellten Guts abhängig ist,

- der Erklärungs- und Beratungsbedarf der Konsumenten, welcher durch die Standardisierung der Ware und ihre Bekanntheit bestimmt wird, sowie

- die Beziehung des Konsumenten zum jeweiligen Produkt, welche nach Gattiker (2001) von der Bedeutung der Eigenschaften der Ware für den Konsumenten abhängt.

Besonders geeignet sind demnach Standard- und Markenartikel sowie digitale bzw. digitalisierbare Produkte. Da es keine Legaldefinition gibt, was unter Innenstadt- bzw.

\footnotetext{
${ }^{11} 55 \%$ der Befragten einer Online-Erhebung des Instituts ibi research der Universität Regensburg gaben an, dass für sie die Produktart ausschlaggebend für den Nichtkauf im Internet ist. Sie halten manche Produkte offensichtlich für nicht geeignet bzw. versprechen sich keinen Mehrwert durch den Online-Kauf (Schneider/Wittmann/Stahl et al. 2012: 34).
}

Zentrenrelevanz von Sortimenten zu verstehen ist (Pangels 2012: 298), und daher keine einheitlichen Sortimentsabgrenzungslisten zur Bestimmung der Innenstadtrelevanz vorliegen, werden im Folgenden die von der IHK Trier (Schmitt 2010: 1 f.) als innenstadtrelevantes Sortiment klassifizierten Produktgruppen hinsichtlich ihrer Eignung für den Online-Handel überprüft. Dazu werden sie entsprechend der Eignungskriterien innerhalb einer Matrix eingeordnet. $^{12}$

Die Abbildung veranschaulicht, dass sich die deutliche Mehrheit des innerstädtischen Sortiments sehr gut und ein insgesamt großer Teil bedingt gut für den Online-Handel eignen. Lediglich zwei Produktgruppen - Antiquitäten und Musikinstrumente - eignen sich aufgrund der hohen emotionalen Beziehung zum Produkt sowie des hohen Erklärungsbedarfes und der komplexen Logistik weniger für den Online-Handel. Das heißt aber nicht, dass der Online-Handel in diesen Produktgruppen unmöglich wäre bzw. nicht praktiziert wird.

\footnotetext{
${ }^{12}$ Manche Zuordnungen sind nicht ganz einfach. Zu Schuhen und Bekleidung haben viele Konsumenten eine hohe emotionale Bindung. Dies spricht für eine bedingte Eignung. Die Umsätze sprechen eine andere Sprache; insbesondere der Anteil des Online-Umsatzes mit Bekleidung am Gesamteinzelhandelsumsatz ist hoch (s. Abbildung 4). Dies dürfte darauf zurückzuführen sein, dass es sich vielfach um Markenartikel handelt, die den Erklärungsbedarf vermindern.
} 
Abb. 4 Anteil des OnlineHandels am Umsatz einzelner Sortimentsbereiche im Jahr 2013. (Quelle: Eigene Darstellung auf Basis von IFH Köln 2014a: 12 und IFH Köln 2014b)

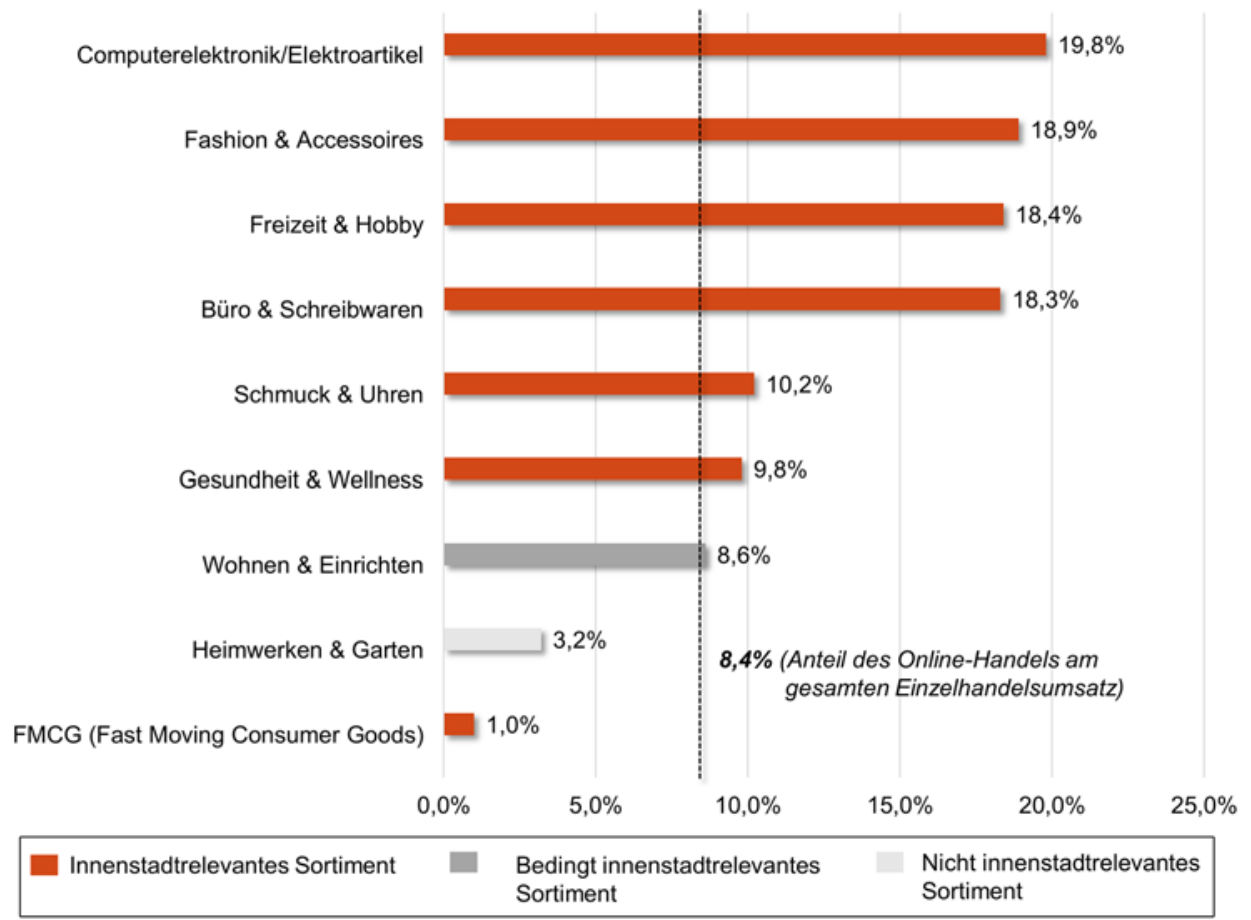

Die hohe Internetaffinität des innerstädtischen Sortiments spiegelt sich in den jeweiligen Anteilen des Online-Handels am Umsatz der innenstadtrelevanten Sortimentsbereiche wider (s. Abbildung 4).

Die Anteile der Sortimentsbereiche liegen, mit Ausnahme der „Fast Moving Consumer Goods“ (FMCG), ${ }^{13}$ deutlich höher - anteilig überwiegend mehr als doppelt so hoch als der durchschnittliche Anteil des Online-Handels am Gesamteinzelhandelsumsatz von 8,4\%. Nicht innenstadtrelevante Sortimente sowie FMCGs haben bisher lediglich einen marginalen Umsatzanteil.

Innenstadtrelevante Sortimente werden folglich gemessen an den Umsatzanteilen - überdurchschnittlich stark über das Internet vertrieben. Dies setzt den innerstädtischen Einzelhandel unter Druck, da langfristig Umsatzeinbußen der Fall sein werden, insbesondere in den benachteiligten Städten (s. Kapitel 4.1).

\section{Blick in die Zukunft - mögliche Konsequenzen für die innerstädtischen Einzelhandelslagen}

Unter der Prämisse weiter steigender Umsatzverschiebungen innerstadtrelevanter Sortimente ins Internet ist von tief greifenden Veränderungen in den Innenstädten auszugehen. Folgende Auswirkungen auf die stationären Geschäfte sind zu erwarten bzw. zeichnen sich bereits heute ab:

\footnotetext{
${ }^{13}$ Dazu zählen Konsumgüter des täglichen Bedarfs wie Nahrungsmittel, Körperpflegeprodukte, Reinigungsmittel usw.
}

- sinkende Umsätze (s. Kapitel 3.2)

- zunehmende Ladenschließungen ${ }^{14}$

- Verkleinerungen der Verkaufsflächen in Folge des sich abzeichnenden Wandels von Verkaufs- zu Ausstellungsflächen ${ }^{15}$

- Zunahme des Hoch- und des Niedrigpreissegments zulasten des mittleren Preissegments (Gaiser/Linxweiler/ Brucker 2005: 18)

- zunehmende Fremdbestimmung der Betreiber und Betreiberwechsel $^{16}$

- neuer Betriebstypenbesatz ${ }^{17}$

\footnotetext{
${ }^{14}$ Laut IFH Köln werden $30 \%$ aller stationären Geschäfte innerhalb der nächsten vier Jahre aus dem Markt ausscheiden (2014c).

${ }^{15}$ Der sich abzeichnende Wandel lässt eine tendenziell sinkende Flächennachfrage vermuten, da weniger Lagerflächen für den Verkauf von Waren vorgehalten werden müssen. Belegt wird diese Vermutung durch die Vermietungsabschlüsse im Jahr 2015. Während die Nachfrage nach Großflächen ab $1.000 \mathrm{~m} 2$ um vier Prozentpunkte im Vergleich zum Vorjahr sank und Großflächen damit $13 \%$ aller Vermietungsabschlüsse ausmachten, konnte bei den kleineren Flächen unter $100 \mathrm{~m} 2$ ein Anstieg auf $24 \%$ verzeichnet werden (Jones Lang Lasalle GmbH 2016: 3).

${ }^{16}$ Die Handelsbetriebe werden selbst zum Handelsgut. „Investoren halten Gebäude in den Städten mittlerweile kaum länger als vier bis sechs Jahre, ehe sie diese wieder verkaufen. Früher war es doppelt so lange. Statt Entwicklung zählen schnelle Wertsteigerung, Vermietung - und der rechtzeitige Absprung" (Böttcher 2013: 114). Vor dem Hintergrund der aktuellen Rahmenbedingungen ist davon auszugehen, dass sich diese Entwicklung zukünftig eher noch verstärken als abschwächen wird.

${ }^{17}$ Zukünftig ist von einer weiteren Etablierung neuer Betriebstypen in den Innenstädten auszugehen. Relativ neu sind die sogenannten „Mono-Label-Stores“, in denen Herstellermarken in eigener Regie
} 
- innovative Ladenkonzepte ${ }^{18}$

Diese betriebsspezifischen Konsequenzen bleiben natürlich nicht ohne Folgen für den Raum. Im schlimmsten Fall kommt es zu „Trading-Down“-Effekten, die ganze Straßenzüge abwerten können. Es ist jedoch davon auszugehen, dass die innerstädtischen Einzelhandelslagen, abhängig vom Innenstadttyp, unterschiedlich stark betroffen sein werden.

So unterscheiden sich Innenstädte, trotz ihrer grundlegenden Gemeinsamkeiten, hinsichtlich ihrer geografischen Lage, ihrer räumlichen, funktionalen, ${ }^{19}$ energetischen ${ }^{20}$ und urbanen $^{21}$ Zentralität sowie in Bezug auf die strukturellen Gegebenheiten wie Stadtgröße, Bevölkerungsentwicklung, wirtschaftliche Situation und Arbeitsmarkt der Stadt, der Mobilität der Bevölkerung, der Baustruktur sowie der historischen Bedeutung. Vor diesem Hintergrund wirkt sich der wachsende Online-Handel stärker oder weniger stark auf die jeweiligen innerstädtischen Einzelhandelslagen aus. Da bisher kaum Studien zu den Auswirkungen des wachsenden Online-Handels mit räumlicher Differenzierung der Ergebnisse vorliegen, werden die innerstädtischen Einzelhandelslagen im Folgenden in Bezug auf Umsatzentwicklung, Flächennachfrage, Mietpreisentwicklung, Entwicklung der Anzahl der Geschäfte sowie die Leerstandsentwicklung grob in zwei Kategorien eingeteilt: die „Verlierer“ und die „Gewinner".

\subsection{Die Verlierer}

Insbesondere in Innenstädten räumlich-strukturell benachteiligter Städte ist von sinkenden Kundenfrequenzen und Umsatzeinbrüchen auszugehen. $\mathrm{Zu}$ ihnen zählen strukturschwache Städte, die zumeist durch hohe

vertrieben werden. Auch international agierende Unternehmen sind verstärkt an Einzelhandelsflächen in den Innenstädten interessiert. Zudem denken immer mehr Online-Händler über die Expansion ihrer Online-Shops in den stationären Handel nach (Range 2013: 36).

${ }^{18}$ Konkurrenzfähig werden diejenigen Betriebsformen sein, die ihre Ladenkonzepte den Bedürfnissen der Konsumenten anpassen. Es ist daher von einer Zunahme innovativer Ladenkonzepte auszugehen, welche die digitalen Medien aktiv für sich nutzen und in den Verkaufsvorgang mit einbeziehen (GfK 2015: 13).

${ }^{19}$ Die Innenstadt ist das funktionale Zentrum einer Stadt. In ihr konzentrieren sich die zentralen öffentlichen Einrichtungen, einzelhandelsnahe Dienstleister, Einzelhandel sowie Gastronomie, welche die zentralen Güter wie Waren, Dienste und Informationen anbieten (Heineberg 2000: 160).

${ }^{20}$ Die Innenstadt ist das energetische Zentrum der Stadt, welches durch seine öffentlichen Räume einen sozialen und kulturellen Austausch ermöglicht und deren wirtschaftliche Kraft aus dem Handel und der Nutzungsvielfalt resultiert (Merk 2011: 15).

${ }^{21}$ "Die dicht bebaute, meist etwas unübersichtliche Innenstadt bietet immer wieder Anregungen und Überraschungen, sie macht neugierig auf explorative Streifzüge in vielfältige Konsum- und Erlebniswelten, (...)“ (Kunzmann 2012: 160).
Sozialausgaben bei gleichzeitig niedrigen Einnahmen, abnehmender Standortattraktivität, verfallenden Infrastrukturen (Deutscher Städtetag 2014: 13) und abnehmender Bevölkerung gekennzeichnet sind sowie großer Konkurrenz zu benachbarten Städten ausgesetzt sind. Zudem unterliegt die kommunale Politik dieser Städte einer starken Abhängigkeit von überlokalen Vorgaben wie staatlichen Transferleistungen von Bund und Ländern sowie Entscheidungen benachbarter Kommunen und hat dementsprechend geringe Handlungsspielräume (Bernt/Weck 2013: 256).

Innenstädte dieser Städte haben aufgrund der negativen Rahmenbedingungen per se eine geringere Aufenthaltsqualität und Attraktivität als Einzelhandelsstandort. Es fehlt an Investitionen in attraktive öffentliche Räume, die Vielfalt an verschiedenen Geschäften ist gering und das Sortiment nicht breit genug, um die Bedürfnisse der heutigen Konsumenten befriedigen zu können. Demgegenüber steht das räumlich und zeitlich unbeschränkt und bequem erreichbare sowie nahezu unbegrenzte Sortiment des Online-Handels, mit dem Einzelhändler in benachteiligten Kommunen nicht konkurrieren können. Es besteht daher besonders in diesen Städten die Gefahr, dass zukünftig noch mehr Kunden verloren gehen.

Schon jetzt beobachten $60 \%$ der vom Forschungsinstitut ECC Köln befragten innerstädtischen klein- und mittelständischen Einzelhändler, dass die Kundenfrequenz zurückgeht (Wilhelm 2014). Nach einer Umfrage des IFH Köln haben bereits heute ca. ein Drittel der Verbraucher die Anzahl der Fahrten in das Stadtzentrum aufgrund des Online-Handels reduziert (Deus 2014). Auch die zukünftige Entwicklung beurteilen die Einzelhändler mehrheitlich negativ. In der Frühjahrsbefragung 2014 des HDE (2014b: 13) gaben $57 \%$ der Einzelhändler an, dass sie für die nächsten 2 Jahre mit sinkenden bis deutlich sinkenden Kundenfrequenzen an ihren Standorten rechnen. Leider wird in den letzten beiden Untersuchungen keine Unterscheidung nach Stadttypus vorgenommen. Es ist jedoch davon auszugehen, dass die Einschätzungen zur zukünftigen Kundenfrequenz in räumlich-strukturell benachteiligten Städten deutlich schlechter waren als in den begünstigten bzw. dass der Rückgang an Fahrten in die Innenstadt vor allem in den benachteiligten Städten zu beobachten ist. Dafür spricht auch das Investitionsverhalten vieler Einzelhändler und Betreiber. Sie schätzen das Umsatzpotenzial insbesondere in kleinen und mittleren Städten zu gering ein, als dass sie zukünftig in solche Standorte investieren würden (Reink 2014: 18). Ein Beispiel dafür ist der Wohnaccessoires-Anbieter Butlers. Laut Aussage des Gründers, Herrn Josten, eröffnet Butlers keine neuen Filialen in Städten unter 100.000 Einwohnern mehr, sondern investiert lieber in seinen Online-Shop (Bender 2013).

Die negative Beurteilung der Entwicklung des Marktpotenzials in benachteiligten Städten schlägt sich auch 
auf die Mietentwicklung nieder. Sie ist in räumlich und strukturell benachteiligten Kommunen entgegen dem allgemeinen Trend negativ. Selbst in den im Allgemeinen stark nachgefragten 1A-Lagen geht das Mietniveau zurück. Zudem stehen vermehrt Ladenlokale ehemals inhabergeführter Fachgeschäfte leer (BMVBS 2011: 19). Je länger und je mehr Ladenlokale leer stehen $-d$. h. wenn strukturelle Leerstände vorliegen -, desto schwerwiegender sind die Folgen. Der Branchenmix und das Image, welche die Attraktivität des Einkaufsangebotes und damit auch der Innenstadt garantieren, werden nachhaltig gestört. Es kommt zur Verödung ganzer Straßenzüge, da auch benachbarte Ladenlokale hinsichtlich Frequentierung und Umsatz beeinträchtigt werden. Zudem verändert sich das soziale Leben in den Innenstädten, da die von Leerstand betroffenen Straßen deutlich seltener besucht und hinsichtlich Gestaltung und Sauberkeit vernachlässigt sowie die leerstehenden Ladenlokale häufig Opfer von Vandalismus werden (Mandac 2011: 11; Wiese-von Ofen 2012: 11).

In räumlich und strukturell benachteiligten Städten mit hohen Umsatzverschiebungen in Richtung Online-Handel und den damit verbundenen Folgen (abnehmende Kundenfrequenzen, Geschäftsschließungen und Leerstände) wird die funktionale Stabilität der Innenstädte allem Anschein nach ins Wanken geraten. Ob der innerstädtische Einzelhandel in diesen Städten weiterhin eine der wichtigsten Leitfunktionen bleibt, ist fraglich. Es besteht daher großer Handlungsbedarf auf Seiten der Stadtplanung und -entwicklung.

\subsection{Die Gewinner}

$\mathrm{Zu}$ den Gewinnern werden die Innenstadtlagen zählen, welche in räumlich-strukturell begünstigen Städten liegen. Räumlich-strukturell begünstigt sind zum einen prosperierende (Groß-)Städte mit Bevölkerungswachstum, die in der Regel höchstzentrale Versorgungsfunktionen übernehmen, sowie zum anderen Mittelstädte in ländlichen Räumen, welche aufgrund ihrer Zentralität von großer Attraktivität für das Umland sind. Die innerstädtischen Einzelhandelslagen in diesen beiden Stadttypen haben durch ihre Versorgungsfunktion für das Umland, welche sich unter anderem in einer höheren Angebotsvielfalt und der damit verbundenen höheren Aufenthalts- und Erlebnisqualität widerspiegelt, per se eine höhere Anziehungskraft als innerstädtische Einzelhandelslagen in benachteiligten Städten (s. Kapitel 4.1).

Vor allem (Groß-)Städte, insbesondere wenn sie viele touristische Attraktionen aufweisen, sind weitgehend immun gegenüber den negativen Auswirkungen des OnlineHandels, da sie über eine ausreichende Nachfrage verfügen. So weisen die einwohnerstärksten Kreise in Deutschland wie in den Vorjahren die höchsten Umsatzsummen auf.
In den zehn einwohnerstärksten Kreisen wurden 2014 $17,5 \%$ des gesamten stationären Einzelhandelsumsatzes in Deutschland erwirtschaftet (GfK 2014: 2).

Generell hält die Nachfrage nach Einzelhandelsflächen, bedingt durch die große Attraktivität des deutschen Einzelhandelsmarktes insbesondere für internationale Einzelhändler, auf hohem Niveau an (Hahn Gruppe 2013: 90). Dies schlägt sich auf die Mietpreisentwicklung nieder. Die Mietpreise von Handelsimmobilien haben sich insbesondere in den Großstädten deutlich erhöht ${ }^{22}$ (Deschermeier/Seipelt/ Voigtländer 2014: 7). Besonders hoch ist der Flächendruck in den 1A-Lagen, der durch die steigende Nachfrage nach "Core-Flächen“ - moderne Flächen, die den heutigen Anforderungen des Einzelhandels genügen - weiter vorangetrieben wird. Daher werden die Mieten 2014 aus Sicht von Vierbuchen (2014: 8) überwiegend moderat weiter steigen.

Auch Mittelstädte mit einer hohen Versorgungsfunktion für das Umland sind räumlich-strukturell begünstigt. Sie erzielen gemäß der GfK rechnerisch Pro-Kopf-Umsätze, die doppelt so hoch sind wie der Bundesdurchschnitt (2014: 2 f.). Daran wird auch der Online-Handel vorerst nichts ändern. Denn die begünstigten Städte haben einen Vorteil, sie profitieren von der veränderten Raumwahrnehmung der Konsumenten.

Denn durch die verstärkte Nutzung des virtuellen Raums in Form des Internets, welche den Konsumenten zu einer größeren zeitlichen und räumlichen Flexibilität verhilft, nehmen sie den physischen Raum anders wahr (Hassenpflug/ Tegeder 2004: 171). Er erfährt eine Aufwertung, da seine besonderen Raumqualitäten - die Atmosphäre ${ }^{23}$ und das zentrale Erleben - als unverzichtbar eingestuft werden. Dies kommt insbesondere den Innenstädten in räumlichstrukturell begünstigten Städten zugute. Einerseits ist die Atmosphäre eines urbanen Raumes im Wesentlichen von seiner Belebtheit abhängig. Ist diese - wie in räumlichstrukturell benachteiligten Städten - nicht (mehr) gegeben, sinkt seine Attraktivität. Andererseits besitzen räumlich-strukturell begünstigte Städte durch ihre besonderen Eigenschaften ${ }^{24}$ in Verbindung mit individuellen lokalspezifischen Elementen eine Authentizität, die anziehend wirkt (Tegeder 2004: 118 f.). Diese Authentizität bzw. der hohe

\footnotetext{
${ }^{22}$ Ausnahmen sind die Städte Düsseldorf, Essen und Dresden.

${ }^{23}$ Empirische Erhebungen von Tegeder in zwei mittelgroßen Städten mit hoher Zentralität - Leipzig und Hannover - haben ergeben, dass die Atmosphäre der Stadt insbesondere für „Onliner“, also für diejenigen, die das Internet regelmäßig nutzen, Anlass für den Einkaufsbesuch der Innenstadt ist. Rund $61 \%$ der Befragten gaben die Atmosphäre als Grund an (,Nonliner“', d. h. Internet-nicht-Nutzer: 54\%) (Tegeder 2004: 118).

${ }^{24}$ Zentrale Merkmale von funktionierenden und damit attraktiven Innenstädten sind Zentralität, Bedeutungsüberschuss, Dichte, Nutzungsmischung, Identifikation und hohe Besucherfrequenz (Stepper 2015: 28 ff.). Sie bieten die Möglichkeit zur sinnlichen Wahrnehmung und zur Face-to-Face-Interaktion.
} 
Grad der Identifikation, den eine attraktive Innenstadt innehat, verhilft ihr zu einem Alleinstellungsmerkmal gegenüber konkurrierenden lokalen Einzelhandelsstandorten und auch gegenüber dem Online-Handel.

Zusammenfassend werden Innenstädte räumlichstrukturell begünstigter Städte - selbst wenn einzelne Betriebsformen von einer sinkenden Nachfrage betroffen sein sollten - auch im digitalen Zeitalter zu den zentralen Versorgungsbereichen einer Stadt zählen, da sie eine günstige Ausgangssituation vorfinden. Ihre überregionale Zentralität, die Nutzungsmischung sowie die besondere Atmosphäre in der Innenstadt führen zu vergleichsweise hohen Passantenfrequenzen, von denen die innerstädtischen Einzelhändler profitieren können. Allerdings reicht es nicht, sich allein auf die traditionellen Stärken zu verlassen. Auch in den räumlich und strukturell begünstigten Städten besteht Handlungsbedarf.

\section{Ausblick}

Auch wenn eine unmittelbare Verbindung der Entwicklungen (Polarisierung zwischen starken und schwachen Zentren) zum Online-Handel bislang nicht belegt werden konnte, ist vor dem Hintergrund der dargelegten Entwicklungen davon auszugehen, dass die Anforderungen an die Qualität der innerstädtischen Einzelhandelslagen aufgrund des Online-Handels steigen werden. Wenn sich Innenstädte auch zukünftig durch ein urbanes Flair und damit inhärent eine hohe Frequentierung auszeichnen sollen, um auf diese Weise eine Voraussetzung für die stabile Umsatzentwicklung im stationären Einzelhandel zu schaffen, haben die kommunalen Akteure für die Aufrechterhaltung und gegebenenfalls für die Verbesserung der Aufenthaltsqualität in den innerstädtischen Einzelhandelslagen insbesondere in den räumlich-strukturell benachteiligten Städten Sorge zu tragen. Nicht das Ignorieren der Digitalisierung, sondern vielmehr die Integration digitaler Medien in die innerstädtischen Einzelhandelslagen sollte daher bei derartigen Überlegungen im Zentrum stehen.

Denkbar ist zum einen die Unterstützung von Multikanal-Formaten (multi channel), die den stationären Einzelhandel mit dem Online-Handel verbinden und ihm dadurch ein zweites Standbein sichern. Zum anderen sollten verstärkt einkaufsunterstützende digitale Medien in den innerstädtischen Einzelhandelslagen eingesetzt werden. Sie ermöglichen eine interaktive Information und Beratung der Konsumenten, mobile „Point of Sale“-Lösungen, ${ }^{25}$ Laden-

\footnotetext{
${ }^{25}$ Die Bezeichnung steht für einkaufsunterstützende digitale Medien wie Tablet-PCs und Smartphones, die am Point of Sale, dem Einkaufsort, beispielsweise zur Verkaufsberatung oder für das kassenunabhängige Bezahlen eingesetzt werden.
}

und Produkt-Virtualisierung sowie intelligente Produkte und Umgebungen und schaffen dadurch einen Mehrwert für Konsumenten und Unternehmen. Beides gelingt aber nur, wenn die notwendigen Rahmenbedingungen für die zunehmende Nutzung der neuen digitalen Techniken in den innerstädtischen Einzelhandelslagen geschaffen werden.

Zentrale Bedeutung kommt in diesem Zusammenhang der Verbesserung der Nutzerfreundlichkeit digitaler Endgeräte sowie der Verfügbarkeit und Leistungsfähigkeit stabiler und sicherer Internetverbindungen zu. Bislang hinkt der flächendeckende Ausbau mit Breitbandverbindungen in Deutschland deutlich den Anforderungen der Nutzer - private Haushalte und Unternehmen - sowie den Versprechungen seitens der Politik hinterher. ${ }^{26}$ Einen zusätzlichen Standortvorteil würden innerstädtische Einzelhandelslagen zudem durch den Ausbau eines frei zugänglichen WLAN-Netzes in den Innenstädten erlangen.

Auch die Nutzer haben spezielle Voraussetzungen zu erfüllen. Sie müssen mit den digitalen Medien umgehen können, d. h. über eine gewisse Medienkompetenz verfügen. Weniger als die Hälfte der Bevölkerung, rund $41 \%$, können laut dem Deutschen Institut für Vertrauen und Sicherheit im Internet (DIVSI) ${ }^{27}$ digitale Medien bislang den eigenen Bedürfnissen und Anforderungen entsprechend nutzen und mit ihnen selbstsicher umgehen (2012: 34 ff.). Dies ist eine Erklärung dafür, warum bislang nur etwas mehr als die Hälfte der Bevölkerung Online-Shopping nutzt (s. Kapitel 3.1), einkaufsunterstützende Medien im lokalen Einzelhandel erst am Anfang stehen sowie Multi-ChannelHandel für die Mehrheit der stationären Einzelhändler bisher nicht infrage kommt (HDE 2013). Es gilt daher, sowohl den Einzelhändlern als auch den Konsumenten Hilfestellungen zu geben. Wichtig sind insbesondere die Vermittlung von Orientierungswissen darüber, was technisch möglich ist, sowie die technische und organisatorische Qualifikation der Nutzer. Weiterhin sollte denjenigen stationären Händlern, die Interesse am Multikanal-Handel haben und zudem über das digitale, organisatorische und finanzielle Potenzial verfügen, entsprechende Unterstützungsangebote gemacht werden. Vor allem kleine und mittelständische Unternehmen haben Nachholbedarf beim Einstieg in das ,Multi-ChannelRetailing“. Sechs von zehn Händlern haben keinen Online-

\footnotetext{
${ }^{26}$ Bis zum Jahr 2014 sollten laut Bundesregierung $75 \%$ der deutschen Haushalte mit Bandbreiten von $\geq 50 \mathrm{Mbit} / \mathrm{s}$ ausgestattet sein (Goldmedia GmbH Strategy Consulting 2013: 2). Die Breitbandverfügbarkeit von Hochleistungsanschlüssen lag Mitte 2014 im bundesdeutschen Durchschnitt jedoch erst bei $64,1 \%$ und in den ländlichen Räumen sogar lediglich bei 20,5\% (TÜV Rheinland 2014: 4).

${ }^{27}$ Das DIVSI unterscheidet zwischen drei Segmenten von InternetMilieus: „Digital Outsiders“, „Digital Immigrants“ und „Digital Natives". Letztere entsprechen den hier genannten $41 \%$ der Bevölkerung. Für sie stellt das Internet einen erweiterten Lebensraum dar, in dem sie sich aufgrund ihrer ausgeprägten IT-Kompetenz sicher und gezielt bewegen (2012: 58).
} 
shop und wollen in naher Zukunft auch keinen eröffnen (ECC Köln 2014: 4).

Die wachsende Bedeutung des Online-Handels und das gleichzeitige Bestehen des innerstädtischen Einzelhandels ist folglich nur ein vermeintlicher Widerspruch. Die Digitalisierung im Allgemeinen und der Online-Handel im Besonderen bieten neben den nicht zu bestreitenden Risiken durchaus Chancen, die Innenstadt langfristig als zentralen Versorgungsbereich einer Stadt zu erhalten. Die Chancen und Risiken sind jedoch ungleich verteilt. Um den kommunalen Akteuren individuelle Handlungsempfehlungen geben zu können, ist es unerlässlich, empirische Forschungen zur Differenzierung der Auswirkungen des wachsenden OnlineHandels nach Standortlagen und Stadttypen zu forcieren.

\section{Literatur}

AFP - Agence France-Presse GmbH (2014): Umsatz im Einzelhandel wird bis 2020 stark sinken. http://www.handelsblatt.com/unternehmen/handel-konsumgueter/studie-umsatz-im-einzelhandelwird-bis-2020-stark-sinken/9730122.html (15.02.2016).

Alby, T. (2008): Web 2.0. Konzepte, Anwendungen, Technologien. München.

ARD/ZDF-Medienkommission (2015): Onlinezugang. http://www. ard-zdf-onlinestudie.de/index.php?id=500 (25.03.2015).

BBR - Bundesamt für Bauwesen und Raumordnung (2010): IRBLagetypengemäßklassischerZentrenkonzeption.http://www.bbsr. bund.de/BBSR/DE/Raumbeobachtung/UeberRaumbeobachtung/ Komponenten/VergleichendeStadtbeobachtung/Raeumliches/ LagetypenIRB/Lagetypen_gro\%C3\%9F.jpg?_blob=normal $\& \mathrm{~V}=1(24.02 .2016)$.

Bender, H. (2013): „Klein- und Mittelstädten droht die Verödung“. https://www.derhandel.de/news/unternehmen/pages/ Handelsimmobilien-Klein--und-Mittelstaedten-droht-dieVeroedung-9524.html (25.03.2015).

Bernt, M.; Weck, S. (2013): Peripherisierung, Schrumpfung und Governance: Handlungsansätze der Stadtpolitik in sechs deutschen Mittelstädten. In: Kuhlmann, S.; Haus, M. (Hrsg.): Lokale Politik und Verwaltung im Zeichen der Krise. Wiesbaden, 256-273.

bevh - Bundesverband E-Commerce und Versandhandel Deutschland e. V. (2015): Bewegtes Jahr 2014 für Online- und Versandhandel. http://www.bevh.org/presse/pressemitteilungen/details/ artikel/bewegtes-jahr-2014-fuer-online-und-versandhandel/ (23.01.2016).

BMVBS - Bundesministerium für Verkehr, Bau und Stadtentwicklung (2011): Weißbuch Innenstadt. Starke Zentren für unsere Städte und Gemeinden. Berlin/Bonn.

Böttcher, D. (2013): Neue Ideen für alte Marktplätze. In: brand eins. Wirtschaftsmagazin, 04, 112-119.

Christ, W. (2014): Konsumkultur und Raumstruktur. Aktuelle Entwicklungen in den USA und Großbritannien. In: Informationen zur Raumentwicklung, 1/2014, 67-80.

Deschermeier, P.; Seipelt, B.; Voigtländer, M. (2014): Mietpreisentwicklung von Gewerbeimmobilien in deutschen Großstädten. In: IW-Trends - Vierteljahresschrift zur empirischen Wirtschaftsforschung aus dem Institut der deutschen Wirtschaft Köln, 41, $3,1-15$.

Deus, R. (2014): Der Fachhandel hat weiter eine Zukunft. http://www. rp-online.de/nrw/staedte/solingen/der-fachhandel-hat-weitereine-zukunft-aid-1.4040110 (23.03.2015).
Deutsche Post DHL (2012): Einkaufen 4.0. Der Einfluss von E-Commerce auf Lebensqualität und Einkaufsverhalten. http:// www.dpdhl.com/content/dam/dpdhl/presse/pdfs_zu_pressemitteilungen/2011_11/dpdhl-studie-einkaufen-4-0.pdf (18.03.2015).

Deutscher Städtetag (2014): Finanzbeziehungen neu regeln, Städte stärken. Schlaglichter aus dem Gemeindefinanzbericht 2014 des Deutschen Städtetages. http://www.staedtetag.de/imperia/md/ content/dst/veroeffentlichungen/gemeindefinanzbericht/2014/ gfb_2014_schlaglichter.pdf (23.01.2016).

DIVSI - Deutsches Institut für Vertrauen und Sicherheit im Internet (2012): DIVSI Milieu-Studie zu Vertrauen und Sicherheit im Internet. Eine Grundlagenstudie des SINUS-Instituts Heidelberg im Auftrag des Deutschen Instituts für Vertrauen und Sicherheit im Internet (DIVSI). Hamburg. https://www.divsi.de/wp-content/ uploads/2013/07/DIVSI-Milieu-Studie_Gesamtfassung.pdf (22.02.2016).

Doplbauer, G. (2015): Ecommerce: Wachstum ohne Grenzen? Online-Anteile der Sortimente - heute und morgen. http:// www.gfk-geomarketing.de/fileadmin/gfkgeomarketing/ de/beratung/20150723_GfK-eCommerce-Studie_fin.pdf (22.01.2016).

Dorn, N. (2015): Vom E-Commerce lernen. RFID und Smartphone als Konversionsoptimierer im stationären Modehandel. Hamburg.

Ebert, O.; Zlonicky, P. (1990): Entwicklung der Innenstädte. In: Bundesforschungsanstalt für Landeskunde und Raumordnung (Hrsg.): Städtebauliche Forschungsaufgaben in den 90er Jahren. Dokumentation eines wissenschaftlichen Seminars des Bundesministeriums für Raumordnung, Bauwesen und Städtebau und der Bundesforschungsanstalt für Landeskunde und Raumordnung am 26./27. Oktober 1989 in Bonn. Bonn, 85-114. = Schriftenreihe „Forschung“ des Bundesministers für Raumordnung, Bauwesen und Städtebau 479.

ECC Köln - E-Commerce Center (2014): Handel im Mittelstand: Wohin geht die Reise? Köln.

EHI Retail Institute GmbH (2015): Whitepaper. Expansionstrends 2015. Köln.

Eli, M.; Laumer, H. (1969): Der Versandhandel. Struktur und Wachstum im internationalen Vergleich. Berlin, München. = Struktur und Wachstum. Reihe Absatzwirtschaft 1.

Gattiker, U. E. (2001): The Internet as a diverse community. Cultural, organizational, and political issues. Mahwah, N.J.

Gaiser, B.; Linxweiler, R.; Brucker, V. (2005): Praxisorientierte Markteinführung. Neue Strategien, innovative Instrumente und aktuelle Fallstudien. Wiesbaden.

GfK (2014): GfK: Einzelhandel wächst 2014 nur durch Online stationäre Umsätze erstmals leicht rückläufig. http://www. gfk-geomarketing.de/fileadmin/newsletter/pressemitteilung/PM einzelhandelsumsatz-2014_de.html (24.01.2016).

Goldmedia GmbH Strategy Consulting (2013): Dritter Monitoringbericht zur Breitbandstrategie der Bundesregierung. Zusammenfassung. http://www.bmvi.de/SharedDocs/DE/Anlage/ Digitales/breitbandstrategie-dritter-monitoringbericht.pdf? blob=publicationFile (08.02.2016).

Hahn Gruppe (2013): Retail Real Estate Report Germany. 8. Ausgabe 2013/2014. Bonn.

Hassenpflug, D.; Tegeder, G. (2004): Städtischer Einzelhandel in vernetzten Zeiten. Forschungsbericht des Projekts „E-commerce and urban trade“. Weimar.

HDE - Handelsverband Deutschland (2013): Onlineshops des stationären Handels. http://www.einzelhandel.de/index.php/ presse/zahlenfaktengrafiken/item/122998-onlineshops-desstation\%C3\%A4ren-handels (08.02.2016).

HDE - Handelsverband Deutschland (2014a): Branchenreport Einzelhandel. Stadt und Handel. http://www.einzelhandel.de/ images/publikationen/Branchenreport_HDE_Stadt_Handel.pdf (17.01.2016). 
HDE - Handelsverband Deutschland (2014b): HDE-Frühjahrs-Pressekonferenz. http://www.einzelhandel.de/images/presse/reden/ Grafiken_Konjunkturumfrage.pdf (23.03.2015).

HDE - Handelsverband Deutschland (2015a): Branchenreport Einzelhandel. Der Handel als Wirtschaftsfaktor. http://www. einzelhandel.de/images/publikationen/Branchenreport-Wirtschaftsfaktor-Handel 2016.pdf (17.01.2016).

HDE - Handelsverband Deutschland (2015b): E-Commerce-Umsätze. http://www.einzelhandel.de/index.php/presse/zahlenfaktengrafiken/item/110185-e-commerce-umsaetze (23.01.2016).

HDE - Handelsverband Deutschland (2015c): Der deutsche Einzelhandel. http:/www.einzelhandel.de/images/presse/Graphiken/ DerEinzelhandelJan2014.pdf (31.01.2016).

Heineberg, H. (2000): Stadtgeographie. Paderborn/München/Wien/ Zürich. $=$ Grundriß Allgemeine Geographie.

Heitfeld-Hagelgans, E. (2012): Das übergeordnete Interesse einer ausgewogenen Einzelhandelsentwicklung in Stadt und Region auf Länderebene (West). In: Kruse, S. (Hrsg.): Handbuch Einzelhandel. Bonn, 19-26.

Hotzan, J. (2004): DTV-Atlas Stadt. Von den ersten Gründungen bis zur modernen Stadtplanung. München.

IFH Köln - Institut für Handelsforschung GmbH (2014a): Branchenreport Online-Handel Jahrgang 2014. Köln.

IFH Köln - Institut für Handelsforschung GmbH (2014b): IFH-Studie: Online-Handel knackt die 40-Milliarden-Grenze - weiterhin zweistelliges Umsatzwachstum. http://www.ifhkoeln.de/pressemitteilungen/details/ifh-studie-online-handel-knackt-die-40milliarden-grenze-weiterhin-zweistelligesumsatzwachstum/ (19.03.2015)

IFH Köln - Institut für Handelsforschung GmbH (2014c): Der Handel muss sich neu erfinden -5 Thesen zur Zukunft des Handels. http://www.ifhkoeln.de/pressemitteilungen/details/der-handelmuss-sich-neu-erfinden-5-thesen-zur-zukunft-des-handels\# (23.01.2016)

IFH Köln - Institut für Handelsforschung GmbH (2015a): OnlineHandels-Umsätze in Deutschland: Von Stagnation keine Spur. http://www.ifhkoeln.de/pressemitteilungen/details/onlinehandels-umsaetze-in-deutschland-von-stagnation-keine-spur/ (23.01.2016)

IFH Köln - Institut für Handelsforschung GmbH (2015b): Umfrage: Schulnote 3 plus für deutsche Innenstädte. http://www.ifhkoeln. de/pressemitteilungen/details/umfrage-schulnote-3-plus-fuerdeutsche-innenstaedte (13.03.2015).

IFH Retail Consultants GmbH (2012): E-Commerce. http://www. handelswissen.de/data/themen/Marktpositionierung/Betriebsform/Distanzhandel/E-Commerce (19.03.2015).

InterOne $\mathrm{GmbH}$ (2011): The Retail Revolution. How digital technologies change the way we shop. http://retail-revolution.interone. de/de/pdf/The Retail Revolution DE.pdf (19.03.2015).

Jones Lang Lasalle $\mathrm{Gmb} H$ (2016): Einzelhandelsmarktüberblick. Deutschland. 4. Quartal 2015. http://www.jll.de/germany/ de-de/Research/Einzelhandelsmarktueberblick-Germany-JLL. pdf?83d8bd22-a0b5-412c-af6d-3b95405a5397 (23.01.2016).

Köcher, R. (2012): ACTA 2012. Handel 3.0. Dynamische Veränderung des Handels. http://www.ifd-allensbach.de/fileadmin/ ACTA/ACTA_Praesentationen/2012/ACTA2012_Koecher.pdf (18.03.2015).

KPMG AG Wirtschaftsprüfungsgesellschaft; EHI Retail Institute (2012): Consumer Markets. Trends im Handel 2020. https:// www.kpmg.de/docs/20120418-Trends-im-Handel-2020.pdf (03.02.2016)

Kunzmann, K. R. (2012): Das urbane Pentagon von Konsum, Tourismus, Kultur, Wissen und Kreativwirtschaft. In: Brake, K.; Herfert, G. (Hrsg.): Reurbanisierung. Materialität und Diskurs in Deutschland. Wiesbaden, 151-163.
Laux, H. D. (2012): Deutschland im demographischen Wandel. Prozesse, Ursachen, Herausforderungen. In: Geographische Rundschau, 64, 7-8, 38-44.

Malaka, R.; Butz, A.; Hussmann, H. (2009): Medieninformatik. Eine Einführung. München.

Mandac, L. (2011): Handel als prägender Faktor der Innenstadt. In: Der Städtetag, 64, 2, 10-12.

Mensing, M. S. (2010): Das Produkt muss stimmen. Welchen Einzelhandel lieben die Touristen? In: cima direkt. Zeitschrift für Stadtentwicklung und Marketing, 4, 30-31.

Merk, E. (2011): Die Innenstadt als Zukunftsgenerator? In: Der Städtetag, 64, 2, 15-17.

Müller-Hagedorn, L. (1998): Der Handel. Stuttgart.

Neue Mediengesellschaft Ulm mbH (2014): Hohe Kunst der Statistik. http://heftarchiv.internetworld.de/2014/Ausgabe-08-2014/HoheKunst-der-Statistik (23.01.2016).

Nitt-Drießelmann, D. (2013): Einzelhandel im Wandel. Hamburg. http://www.hwwi.org/fileadmin/hwwi/Publikationen/Partnerpublikationen/HSH/2013_05_23_HSH_HWWI_Einzelhandel. pdf (17.01.2016).

Pangels, R. (2012): Zentrenrelevanz von Sortimenten - Theoretische Annäherung an ein viel diskutiertes Planungsinstrumentarium. In: Kruse, S. (Hrsg.): Handbuch Einzelhandel. Bonn, 294-320.

Range, T. (2013): Wandel lohnt sich. Der Onlinehandel wächst und wächst und wächst - auf Kosten des stationären Handels. Aber das ist kein Naturgesetz. In: brand eins. Wirtschaftsmagazin, 04, 30-36.

Reink, M. (2014): Perspektiven schwacher Zentren. Zusammenhänge von Handels- und Stadtentwicklung. In: RaumPlanung, 176, 6, $17-21$.

Schmitt, M. (2010): Einzelhandel: Abgrenzung zentrenrelevanter von nicht-zentrenrelevanten Sortimenten. http://cms.ihk-trier.de/ ihk-trier/Integrale?MODULE=Frontend. Media\&ACTION= ViewMediaObject\&Media.PK=10367\&Media.Object.ObjectType=full (24.01.2016).

Schneider, C.; Wittmann, G.; Stahl, E.; Weinfurtner, S.; Pur, S. (2012): Multikanalvertrieb: ganz klar! Aber wie? Fakten aus dem deutschen (Online-)Handel. Regensburg. http://www. ecommerce-leitfaden.de/download/studien/Studie_Multikanalvertrieb.pdf (24.01.2016).

Schubert, D. (2008): Die Fußgängerzone. Auslaufmodell oder Beitrag zur Renaissance europäischer Stadtkultur? In: Altrock, U.; Kunze, R.; Petz, U. von; Pahl-Weber, E.; Schubert, D. (Hrsg.): Schwerpunkt „Aufwertung im Stadtumbau“. Beiträge aus Lehre und Forschung an deutschsprachigen Hochschulen. Berlin, 33-54.

Statistisches Bundesamt (2014): 45 Millionen Menschen in Deutschland kaufen über das Internet ein. https://www.destatis.de/DE/ PresseService/Presse/Pressemitteilungen/zdw/2014/PD14_036_ p002pdf.pdf?_blob=publicationFile (24.01.2016).

Stepper, M. (2015): Einkaufsstandort Innenstadt. Qualifizierung innerstädtischer Einzelhandelslagen vor dem Hintergrund des zunehmenden Online-Shopping. Kaiserslautern (Dissertation, Technische Universität Kaiserslautern).

Tegeder, G. (2004): Konsumenten im Netz der Möglichkeiten. Zum Einfluss des Internet auf den städtischen Einzelhandel. In: Zeitschrift für Wirtschaftsgeographie, 48, 1, 111-123.

TÜV Rheinland - TÜV Rheinland Consulting GmbH (2014): Bericht zum Breitbandatlas Mitte 2014 im Auftrag des Bundesministeriums für Verkehr und digitale Infrastruktur (BMVI). Teil 1 - Ergebnisse. http://www.zukunft-breitband.de/SharedDocs/ DE/Anlage/Digitales/bericht-zum-breitbandatlas-mitte-2014-ergebnisse.pdf? blob=publicationFile (08.02.2016).

Vierbuchen, R. (2014): Globaler Vermietungsmarkt. Mieten in den Top-Lagen werden 2014 steigen. http://www.rohmert-medien. de/wp-content/uploads/2014/01/Der-HandelsimmobilienReport-Nr-162.pdf(19.03.2015). 
Weers-Hermanns, T. F. (2007): Planerische Steuerung des Einzelhandels aus kommunaler und regionaler Sicht. Göttingen. = Planungsrecht 12.

Wiese-von Ofen, I. (2012): Einleitende Gedanken zur allgemeinen Bedeutung des Einzelhandels für die Stadtentwicklung. In: Konze, H.; Wolf, M. (Hrsg.): Einzelhandel in Nordrhein-Westfalen planvoll steuern! Hannover, 9-14. = Arbeitsberichte der ARL 2.

Wietzel, I. (2007): Methodische Anforderungen zur Qualifizierung der Stadtplanung für innerstädtisches Wohnen durch Mixedreality-Techniken und immersive Szenarien. Kaiserslautern (Dissertation, Technische Universität Kaiserslautern). $=$ Schriften zur Stadtplanung 7.
Wilhelm, S. (2014): Mittelstand. Händler haben online Nachholbedarf. http://www.derhandel.de/news/technik/pages/\%20MittelstandHaendler-haben-online-Nachholbedarf-10700.html (23.03.2015).

Wolf, M. (2012): Anforderungen an Einzelhandelsgutachten. In: Konze, H.; Wolf, M. (Hrsg.): Einzelhandel in Nordrhein-Westfalen planvoll steuern! Hannover, 114-134. = Arbeitsberichte der ARL 2.

Wüstenrot Stiftung (2008): Fokus Innenstadt. Innenstadtentwicklung in baden-württembergischen Mittelstädten. Ludwigsburg. 